\title{
Natural Selection and Multi-Level Causation
}

\author{
Maximiliano Martínez $z^{\S}$ and Andrés Moya
}

In this paper, using a multilevel approach, we defend the positive role of natural selection in the generation of organismal form. Despite the currently widespread opinion that natural selection only plays a negative role in the evolution of form, we argue, in contrast, that the Darwinian factor is a crucial (but not exclusive) factor in morphological organization. Analyzing some classic arguments, we propose incorporating the notion of 'downward causation' into the concept of 'natural selection.' In our opinion, this kind of causation is fundamental to the operation of selection as a creative evolutionary process.

\section{KEYWORDS \\ Downward causation $\bullet$ Levels of hierarchical organization $\bullet$}

Natural selection $\bullet$ Positive view

\begin{abstract}
Whatever the cause may be of each slight difference in the offspring from their parents [...] it is the steady accumulation, through natural selection, of such differences when beneficial to the individual, that gives rise to all the more important modifications of structure, by which the innumerable beings on the face of this earth are enabled to struggle with each other, and the best adapted to survive.
\end{abstract}

- Darwin (1859, 170)

Novelties come from previously unseen association of old material. To create is to recombine. - Jacob $(1977,1163)$

\section{Introduction}

It is generally held in various fields that, with his work published in 1859, Darwin explains and resolves the issue of the origination of complex biological traits by proposing the process called 'natural selection.' One of Darwin's achievements was to give an answer in strictly naturalistic and mechanistic terms to the riddle of design in nature as opposed to the common theological explanation at that time, advocated mainly by William Paley (Paley 1836 [1802]; cf. Gould 1999; Ruse 1979). Indeed, it is well known that Paley's work greatly influenced Darwin's thinking (Ospovat 1981; Ruse 1979; Gould 1999). This explanation of living design, brought about by a naturalistic phenomenon - natural selection - came to be one of the most successful aspects of the Darwinian revolution. However, in recent decades an important debate about the causal role played by natural selection in shaping the phenotypic traits of organisms has arisen. The debate began with the criticisms made by Karen Neander (1988, 1995a, b) of Elliott Sober's view of natural selection (Sober 1993 [1984], 1995), and has continued since. According to Sober, natural selection explains

\footnotetext{
$\S$ Departamento de Humanidades, Universidad Autónoma Metropolitana-Cuajimalpa, Baja California 200 (5to Piso), DF 06760, Mexico

‡ Unidad Mixta de Investigación en Genómica y Salud CSISP/UVEG and Instituto Cavanilles de Biodiversidad y Biología Evolutiva, Avenida de Cataluña 21, 46020, Valencia, Spain E-mail: mmartinez@,correo.cua.uam.mx
} 
the frequencies of traits in populations, not "why particular individuals have the traits they have" (Sober 1995, 385). Following Sober's reasoning, several authors have argued that selection has no creative role in the origination and shaping of organisms' traits. From this viewpoint, now known as 'the negative view,' natural selection is considered as a merely negative process, fulfilling only the function of filtering and distributing the percentage of variants already existing in a population, which originated and were shaped by other biological means (Endler 1986; Walsh 1998; Ariew 2003; Pust 2004). This negative view opens up a favorable area where other factors present in the origins of organismal form can be considered (e.g., developmental constraints and self-organization, factors that have traditionally been out of focus in evolutionary research), but it poses the risk of ignoring (at least theoretically) a creative role that natural selection could play in this process. The authors that defend this creative role of selection are on the other side of the debate (e.g., Neander 1988, 1995a, b; Matthen 1999, 2003; Forber 2005; Nanay 2005). ${ }^{1}$ The issue at stake is whether or not natural selection plays a creative causal role in the generation of organismal form, or is it merely a filter for unsuccessful forms originating via other biological factors.

The aims of the present paper are twofold. Our first aim is to reinforce the idea that the notion of 'negative natural selection' is too narrow of a concept to adequately capture the role that natural selection plays in evolution. To that end we examine some of the ideas that are behind the debate between negative and positive views of selection (Section 2). Second, we undertake, through a multilevel approach, the formulation of a positive concept of natural selection, which would provide a more far-reaching explanation for the creative potential it holds (Section 3). We look at the works of Dobzhansky, Ayala, Neander, and Campbell, where it is proposed that natural selection has a direct causal influence on the creation of organismal traits. These works provide the basis for our conceptual revision. In conclusion we defend the importance of downward causation as the underlying basis of the creative ability of natural selection.

\section{Positive and Negative Views of Natural Selection}

To understand the concepts of positive and negative natural selection, we mainly draw upon the debate between the philosophers Elliott Sober (1993 [1984], 1995) and Karen Neander (1988, 1995a, b), to which many others have also contributed. ${ }^{2}$ The debate revolves around the role natural selection plays in evolution.

\subsection{Natural selection as a negative factor}

In The Nature of Selection (1993 [1984], 148ff) Sober has persuasively argued that natural selection explains the persistence and distribution of adaptations in populations, emphasizing its distributive causal role. He maintains that in no way does selection explain either the ontogenetic development of adaptive traits, less still their creation or origin. Sober provides an illustrative example of the character of selective explanations (1993 [1984], 149; 1995, 384): imagine a classroom where there are only children who have grade-three reading level proficiency. Now, if we ask ourselves why these children have grade-three reading proficiency, we can respond in two ways. If we consider a developmental explanation, then we must explain how each child in the room gradually developed the ability to read, until reaching grade-three proficiency. If we consider the question of selection, we should find out which criteria were required to allow individuals to be admitted to the room (in this case, having grade-three reading proficiency). The point of this example is clear and simple: evolutionary explanations based on natural selection are of the latter kind, i.e., calling upon selective explanations for evolution focuses on determining the reasons why individuals with a specific adaptive trait thrived and persisted from the past, over individuals who did not have that trait. Thus: "the frequency of traits in a population can be explained by natural selection, even though the possession of those traits by individuals in the population cannot" (Sober 1993 [1984], 152). To explain the origin of a trait in individuals we must explain the processes by which it originated or emerged in terms of mutations and mechanisms of heredity:

Natural selection does not explain why I have an opposable thumb (rather than lack one). This fact falls under the purview of the mechanism of inheritance... There are only two sorts of individual-level facts 
that natural selection may explain. It may account for why particular organisms survive and why they enjoy a particular degree of reproductive success. But phenotypic and genotypic properties of individuals - properties of morphology, physiology and behavioral - fall outside of natural selection's proprietary domain (Sober 1993 [1984], 152).

These ideas have led many authors to see Sober as one of the main proponents of the negative view of natural selection (Endler 1986; Neander 1988, 1995a, b; Matthen 1999, 2003; Pust 2004; Forber 2005; Nanay 2005; McLaughlin [manuscript in preparation]). As Nanay put it: "Sober claims that selection is a negative force: it does not create; it only destroys" (Nanay 2005, 1101). Some points are worth noting here. First, we believe that Sober's argument is correct: selection does explain the frequencies of traits in populations, because it has a distributive causal role in evolution. And it is this distributive role of natural selection that lies at the core of what is known as 'the negative view.' Second, we also think that an overemphasis on this distributive role can promote an unjustified implication, such as that natural selection doesn't have any creative role in the origination and shaping of organismal traits (contrary to what Darwin thought). Beyond Sober's ideas about the role of selection in evolution, it is clear that on the negative view natural selection has an exclusively distributive role. ${ }^{3}$

Numerous authors, both biologists and philosophers, adopt and defend versions that are compatible with the negative view of selection so far presented (Endler 1986; Saunders 1989; Gilbert et al. 1996; Walsh 1998; West-Eberhard 2003; Ariew 2003; Müller 2003; Pust 2004; Kirschner and Gerhart 2005), which takes the discussion beyond the conceptual debate itself. ${ }^{4}$ An exclusive focus on selection's distributive role has had two direct results. First, it has strengthened criticisms that the Modern Synthesis is a partial and incomplete theory of evolution because it does not address the origins and of organismal form. A common approach emerging from this line of thought is that if natural selection is not a causal factor in the generation of organismal form, then it is necessary to have recourse to other areas of biology to explain this fundamental and primary phenomenon (Saunders 1989; Müller and Newman 2003). ${ }^{5}$ Second, this concept of natural selection has spread widely both in specialized and vernacular realms, supported by some intuitive ideas about the way natural selection operates (filtering unsuccessful phenotypes). ${ }^{6}$ To sum up: according to the negative view, natural selection merely explains frequencies of traits in populations, traits that originated and were formed strictly by biological factors other than selection.

\subsection{Natural selection as a creative factor}

From the contrary perspective, many authors have advocated a positive causal role for natural selection in shaping the traits of organisms (Ayala 1970, 1993, 1999; Dobzhansky 1974; Jacob 1977; Dawkins 1986; Neander 1988, 1995a, b; Matthen 1999, 2003; Gould 2002; Forber 2005; Nanay 2005; Godfrey-Smith 2009; McLaughlin [manuscript in preparation]). These authors defend a creative role for selection in evolution that complements its distributive role: selection is a causal factor in the generation and organization of organismal form. To better understand this capacity, we first point to important aspects of Neander's work on cumulative selection and then call attention to some ideas put forward by Dobzhansky.

In order to respond to Sober's view of selection, and in line with Ayala's $(1970,1993)$ reasoning in terms of probabilistic causes, Neander developed an argument to defend the creativity of natural selection based on the idea of the accumulation of successful selective events (1988, 1995a, b). ${ }^{7}$ She distinguishes two types of selection: single-step and cumulative. In a sequence that generates random results and then chooses one of them (Random/Selection sequence), single-step selection may involve multiple repetitions of the sequence, but these are isolated and not interconnected; for example, the tossing of a coin multiple times. By contrast, cumulative selection involves multiple interconnected repetitions. Every new sequence starts on the outcome of the previous one. The results of each selection type differ greatly, due to how each one operates. In single-step selection, the outcome of each Random/Selection sequence is isolated from the rest and does not alter the likely outcome of future sequences. By contrast, in cumulative selection each Random/ Selection sequence influences and alters the possible outcomes of future sequences, as every sequence acts on the results of the previous one (except, of course, the first). The following example, which is a modification of that used by Neander (1995a, 74), illustrates the influence of cumulative selection on the 
probability of the subsequent results of interconnected Random/Selection sequences occurring. Imagine we have a box containing sixteen balls, half of which are numbered from ten to eighty in multiples of ten. At the bottom of the box there are eight holes with the numbers 10, 20,30, 40, 50, 60, 70 and 80, into which, after spinning the box, the respective balls should fall whose numbers match; i.e., the ball with number 10 into the 10th hole, the ball with 20 into the 20th hole, and so on. The probability that all eight balls fall into their respective holes after spinning the box is extremely low. One might spend a lifetime trying again and again without ever succeeding. Now imagine that in the many attempts, each time a ball falls into the right one of the eight holes, it stays there from then on (it does not come out of its hole in the next spin). Thus, after multiple spins, the probability of filling all eight holes with their respective balls will be gradually and significantly increased.

How does this example relate to the process of natural selection? The point is that for Neander natural selection operates as a cumulative process, not as a single step. In nature, a previous selection process can greatly alter the probability of subsequent results (translated into subsequent gene frequencies). This impact of natural selection on the subsequent selection process is made possible through heredity, which can be seen via the following thought experiment (Neander 1995a, 77). Imagine we have three types of genes: $G_{1}, G_{2}$ and $G_{3} . G_{3}$ is slightly better (in adaptive terms) than $G_{2}$ and $G_{2}$ is slightly better than $G_{1}$. $G_{1}$ is genetically closer to $G_{2}$ than to $G_{3}$ while $G_{2}$ is at the same genetic distance from $G_{1}$ and $G_{3}$. Now, if in a population strictly of G1 individuals, one of them mutates to $G_{2}$, causing the $G_{2}$ trait to become established after several generations and displacing $G_{1}$, then natural selection, having selected $G_{2}$ and allowed it to become established due to its higher level of fitness, has greatly increased the likelihood of $\mathrm{G}_{3}$ emerging (for $\mathrm{G}_{3}$ is closer to $G_{2}$ than to $\left.G_{1}\right){ }^{8}$ As Neander says: "If there is a small chance of an individual instance of [ $G_{2}$ ] mutating into $\left[\mathrm{G}_{3}\right]$, the more instances of $\left[\mathrm{G}_{2}\right]$ there are, ceteris paribus, the larger the chance there is of one instance of $\left[\mathrm{G}_{3}\right]$ arising" (Neander 1995a, 77). This reasoning allows her to state that: "Selection does more than merely distribute genotypes and phenotypes ... it plays a crucial role [in] determining which new phenotypes and genotypes will emerge" (Neander 1995b, 585). Thus, in probabilistic terms, natural selection promotes change in the direction of increased adaptive design, provided that this increase implies an increase in the fitness of carriers of the trait. It is in this sense that Neander sees natural selection as a creative force. $^{9}$

\subsection{Dobzhansky's 'channeling' and the non-isotropy of variation}

We hope, thus far, to have shown the important difference between the two approaches to natural selection when providing an explanation for an organism's traits. If we follow the advocates of negative natural selection, then selection is far from playing a causal role in the generation of organismal form. If we subscribe to the contrary proposal, natural selection plays a crucial causal role in generating adapted forms. However, at this point, there is a need to define more precisely the type of causation by which positive natural selection operates creatively. The positive view analyzed so far is focused on the genetic level, but analyzing the individual (or organismal) level now becomes necessary in order to have a better understanding of selection's causal role. As we will see, the organismal level is a key player in the process. Before going on to examine this issue, we would like to dwell on the idea that natural selection is creative in terms of what Dobzhansky put forward in one of his classic works, illustrating to some extent the type of causation to which we will refer in the following section.

In "Chance and Creativity in Evolution" (Dobzhansky 1974), Dobzhansky explains how natural selection is creative through the accumulation of successful variations to impose adaptive evolutionary direction in the formation of the traits of organisms. In our view, one of the most important features of his discussion is the verb used to represent the causal action of natural selection in the process of giving adaptive evolutionary direction to a trait: to channel. Dobzhansky used "channel" to describe the adaptive direction or bias that natural selection imposes on mutation and genetic recombination processes. The directions of a gene mutation are determined by its evolutionary history: "The mutational repertory of a gene is a function of its structure, and hence of the billions of years of its evolution" (Dobzhansky 1974, 315). Moreover, for Dobzhansky, the word 'chance' is misused when talking about the origin of a mutation and its effects. These assertions, in our opinion, have implications for evolutionary theory: variation 
(produced by mutation and recombination) is not isotropic, as it is conditioned by its own history, which, in turn, is conditioned by natural selection. In other words, what Dobzhansky emphasized is that the variation of a gene is guided by its historical burden, which, in turn, has been channeled by natural selection.

[...] it must be stressed that selection puts a restraint on chance and makes evolution directional. Usually, but though not invariably, selection increases the adaptedness of the population to its environments. It is responsible for the internal teleology ... so strikingly apparent in all living beings. The turmoil of mutation and recombination is curbed and channeled in the direction of adaptedness (Dobzhansky 1974, 317 , our emphasis). ${ }^{10}$

Dobzhansky's concept of channeling helps to bring out some important aspects of the causal processes by which natural selection operates. Natural selection operates on the material it has formed previouslymaterial bounded and channeled by the accumulation of past selection events. This fact is what makes natural selection able to direct evolutionary change through the channels it sets itself. It is true that selection can only work with existing material (Jacob 1977). The point here, as stated by Dobzhansky, is that this material is already conditioned, channeled, and constrained by the discriminating and gradual action of past natural selection events. In other words, the accumulation of successful combinations of variations targeted by selection, generation after generation, channels variation itself in specific directions. As is stressed by Matthen (2003), this channeling effect of natural selection is present in Neander's approach. To put it abstractly, the emergence of $G_{3}$ is more probable when natural selection has selected $G_{2}$ over $G_{1}$, because it has channeled this variation. Selection has channeled mutation and recombination events from $G_{1}$ toward $\mathrm{G}_{3}$. We can extend the model forward and imagine a possible $\mathrm{G}_{4}$, a possible $\mathrm{G}_{5}$, etc., and also extend it backwards and imagine a $G_{0}$, a $G_{-1}$, etc. The causal chain that would go from $G_{-1}$ to $G_{5}$ would be possible thanks to the channeling of variation due to cumulative, sequential selection. As Neander $(1988,425)$ puts it: "Causal explanations are elastic creatures. We can stretch the explanans back in time as far as our knowledge permits and as far as is practical." On the other hand, we can also say that, thanks to the selection of specific individuals and their subsequent reproduction, it is possible to implant information of past events of natural selection in an organism's DNA. In other words, through heredity, the history of previous organisms (of past generations) is incorporated in the beginning of every new organism's developmental process. The result of this historical process is then present during an organism's ontogeny.

Even though natural selection can operate at different levels (Okasha 2006), we agree with Mayr (2002), Matthen (2003), and Okasha (2006) that selection operates primarily on organisms, which is the main factor in the evolution of their adaptive traits. "Natural selection leads organisms to evolve adaptations. The existence of organismic adaptations ... shows the importance of organism-level selection in shaping the biota" (Okasha 2006, 12). This is consistent with the analysis so far presented, because the action of selection at the individual level has two interlevel consequences: (a) choosing some individuals over others changes the distribution of individuals in populations because there will be more individuals of the kind that natural selection selected than of the kind it rejected (recall Sober's room example); and, (b) this process changes the configuration of the genetic pool of the population because there will be more genes in it of the individuals that natural selection selected than genes of the individuals that were not (recall Neander's example, above). In this sense, a fact occurring at the organismal level (selection choosing some individuals over others, according to fitness) has effects on both a higher level (characteristics of populations) and a lower level (characteristics of genetic pools). If we focus on the latter relationship (individual and genetic levels), it is possible to see the continuation of the process because the genes that are passed on will be the starting point of the new generation. The organisms that survive and reproduce will pass their genes to the next generation, channeling the kind of genes that will be present subsequently (under most conditions, the genes that come from the fitter organisms). And because this process is cumulative (in Neander's sense), the more generations that pass in a population, the more specific kinds of genes the genetic pool will have. It is this 'steady accumulation' that produces historical channeling of variation (in Dobzhansky's sense), increasing the probability of occurrence of successful mutations and recombination.

In the next section we describe the causal workings of natural selection as a case of downward causation between levels of organization in the sense proposed by D.T. Campbell. In our view, the concept of 
'downward causation' captures and successfully reflects a very important feature of the creative causation present in natural selection.

\section{Natural Selection and Downward Gausation}

We urge that interlevel causation should feature centrally in explanatory hypothesis of evolution. — Vrba \& Eldredge $(1984,146)$

\subsection{Campbell and his causation proposal}

The notion of downward causation has been examined and referred to mainly in discussions about reductionism, emergentism, hierarchical levels of biological organization, evolutionary epistemology, mental supervenience, systems biology, and complex systems theory (Campbell 1974; Popper 1978; Sperry 1986; Kim 1992; Juarrero 1999; El-Hani and Emmeche 2000; Andersen et al. 2000; Bickhard and Campbell 2003; Soto and Sonnenschein 2005; Noble 2006; Bedau 2008; Mitchell 2009). Currently, significant discussion is underway not only with respect to the aforementioned topics but also for the whole conceptual framework of contemporary theoretical biology. This is why it is vital to analyze the relevance of downward causation to the concept of natural selection as a positive causal factor. Although there has been significant progress in consolidating and understanding the phenomenon of downward causation (not without controversy), for our present purposes we will stick to the basic canonical exposition offered by Campbell. Our goal is to link the notion of downward causation, in its original (and therefore clear and robust) formulation with the argument for creative natural selection. For this reason we ignore these discussions surrounding the phenomenology of such causation, hoping to address them in the future in more detail.

The theorist responsible for providing initial guidance for this unorthodox idea of causation was Donald Campbell. His work has become the foundation of many important subsequent works on downward causation (as noted above). Let us see what his analysis entails. Campbell (1974, 180ff) asserts that nature is organized hierarchically into different levels: molecules, cells, tissues, organs, organisms, populations, species and ecosystems. Each of these levels has a factual reality and organizes the actual existing units present in the lower level. He proposes the principle of downward causation to refer to the influence that higher levels exert on lower ones and links it with the operation of natural selection.

[The principle of downward causation]: Where natural selection operates, through life and death at a higher level of organization, the laws of the higher-level selective system determine in part the distribution of lower-level events and substances ... All processes at the lower levels of a hierarchy are restrained by and act in conformity to the laws of the higher levels (Campbell 1974, 180).

Grouping together numerous generations of temporarily successive self-replicating entities is useful for understanding the central point that the concept of downward causation attempts to reflect: the states and events of entities at a higher level exert causal influence (in the future) on their own subsequent replications. This is the way in which generationally successive entities of a higher level establish a causal connection; i.e., through the influence exerted on future lower-level entities, which, in turn, are responsible for the construction of new higher-level entities (in a typical process of upward causation). In this case, Campbell is considering the reciprocal causal relationship between two levels (through a replication process): the organismal (phenotype) and the molecular (genotype).

To make the matter clearer we shall reconstruct the example that Campbell $(1974,181)$ uses to illustrate the action of downward causation and its relationship with natural selection. If we consider the anatomy of the jaws of a worker termite or ant, we note that the ligaments and muscle surface obey the laws of mechanics (Archimedes lever), and are designed optimally to apply maximum force at a determined distance from the joint. This fact agrees with physics, but is different from that implied by molecular processes, which are those that govern the production of specific proteins building the muscle and shell of the mandibular system. Such macro-mechanical laws operate at the level of organisms. Thus, according to Campbell, in 
order to understand and explain the particular and exact distribution of proteins that make up the jaw as well as the corresponding specific DNA sequence, which are at the lower level (molecular), it is necessary to determine the laws of the lever at the higher level (organisms). For Campbell, this is one of the processes that involves both directions of causation, as it is organism-scale natural selection that determines (causes) what specific types of proteins are present at the molecular level, although the immediate microdetermination (cause) is in the direction of DNA $\rightarrow$ protein $\rightarrow$ jaw. Thus the events at a higher level of organization (individuals) partly determine the permanence, formation, and distribution of the organization of entities at lower levels (DNA), which in turn will subsequently reproduce, through upward causation, new higher-level entities.

In other words, the adaptive success or failure of certain higher-level entities has a decisive effect on the future presence and distribution of the lower-level entities that (re)produce them. The adaptive role of ants' jaws determines the continuity or disappearance of the DNA strands that produce them. It is in this sense that higher-level entities, through downward causation, have an impact on future events in both entities of the same level (individuals) and the lower levels that compose them (DNA).

\subsection{Revising the concept of natural selection}

We think that the notion of downward causation proposed by Campbell helps enormously in capturing the positive action of natural selection between levels. Linking this notion of downward causation (which focuses on the organismal level) with the proposed positive selection of Ayala, Neander, and Dobzhansky (which focuses on the genetic level), we can better understand how the selective events that occur at a time $t$ at a higher level determine certain entities that later appear at the lower level, all in a reproductive chain. Natural selection at the individual (higher) level determines which genetic material (lower level) will prevail and which will disappear in the following generation(s). The relationship between downward causation and natural selection is possible thanks to the phenomenon of heredity. It is precisely this type of top-down causation that allows natural selection to determine and channel the material upon which future variation will emerge, where it will again subsequently operate. As noted above, the main selective factor promoting the channeling of the genetic material does not operate directly at the genetic level; it does so at the level of the individual. But the top-down connection between the two levels is possible through downward causation. The selection of specific individuals progressively influences, through downward causation, the composition of the genetic pools, making the appearance of successful genetic combinations more probable. In our view, this fundamental multilevel fact demands a revision of the concept of natural selection; we must incorporate the notion of downward causation into our conception of natural selection. This reformulation would enable us to define more clearly the positive causal role that natural selection plays in the production and pattern that organismal form takes.

In the action of natural selection, operating at a higher level and influencing in turn the future of the lower level material, it is possible discern four central points: (1) the influence of natural selection on future genetic material is exerted through downward causation; (2) this influence orients the channels of evolutionary direction that natural selection imposes upon the material; (3) every selection event operates on the material that natural selection has historically been channeling over successive generations of a lineage; and, (4) by influencing future genetic material (lower level), individuals (higher level) that are made up of such genetic material can, by transitivity, also be influenced.

Having said this, we think it is appropriate to clarify a couple of points regarding our analysis of the relationship between downward causation and natural selection. First, it is necessary to start from the uncontroversial assumption of the existence of real, biological, hierarchically organized levels, and that they are causally related. The second relevant clarification is that the downward causation proposed does not lead to two types of ontologically separate causal actions (upward and downward causation), but rather it draws attention to how a series of the same type of causal events is concatenated as a historical continuum of multiple steps. If we consider a single step, then we observe an upward causal relationship; on the contrary, if we consider a sequence of steps, we will see the action of downward causation. That is, the way of understanding the creativity of natural selection implies understanding its influence, through heredity, on the 
process of upward causation in every generation of individuals. Put differently, the way in which natural selection can be creative is through its influence on the future of upward causation processes initiated by DNA. It is for this reason that we must consider at least two generations to understand how it works (Figure 1). But at the same time one must consider a sequence of many interrelated generations to understand the process of creativity itself and see its cumulative effects - the greater the accumulation, the greater the channeling effect on DNA (Figure 2). Thus, natural selection, through downward causation, exerts its influence from the very moment the process of upward causation in the subsequent generation begins (as in the example given by Campbell), but its cumulative and creative effects are more powerful via the channeling effects that have taken place over a large number of generations, leading up to the construction, in evolutionary time, of an organ as complex as the vertebrate eye (for example). Given that Figure 1 represents a diachronic feedback process (the transition between two generations) that can be iterated and accumulated throughout evolution, the details of this graph can be simplified in order to see how several generations are linked through heredity (Figure 2). If the three sets of arrows in Figure 2 are taken as a causal continuum, it is possible to observe the influence of and channeling produced by a past selective event on any subsequent event. Any point of the continuum is conditioned by its past events and it will condition its future events, due to the channeling effects of downward and upward causation operating together. Thus, genetic, developmental, and selective processes, which occur at different levels of biological organization, are causally connected and determine each other in evolution. Selection affects, in this way, the source of variation.

One must bear in mind that by proposing that biological organization is the result of a feedback process of upward and downward causation between levels, we are simply suggesting the necessity of adopting a multilevel causal approach in order to understand the channeling effect produced by a long succession of events that are historically linked. Our analysis shows the necessity of incorporating the concept of downward causation to explain the causally creative role of natural selection. A process like natural selection

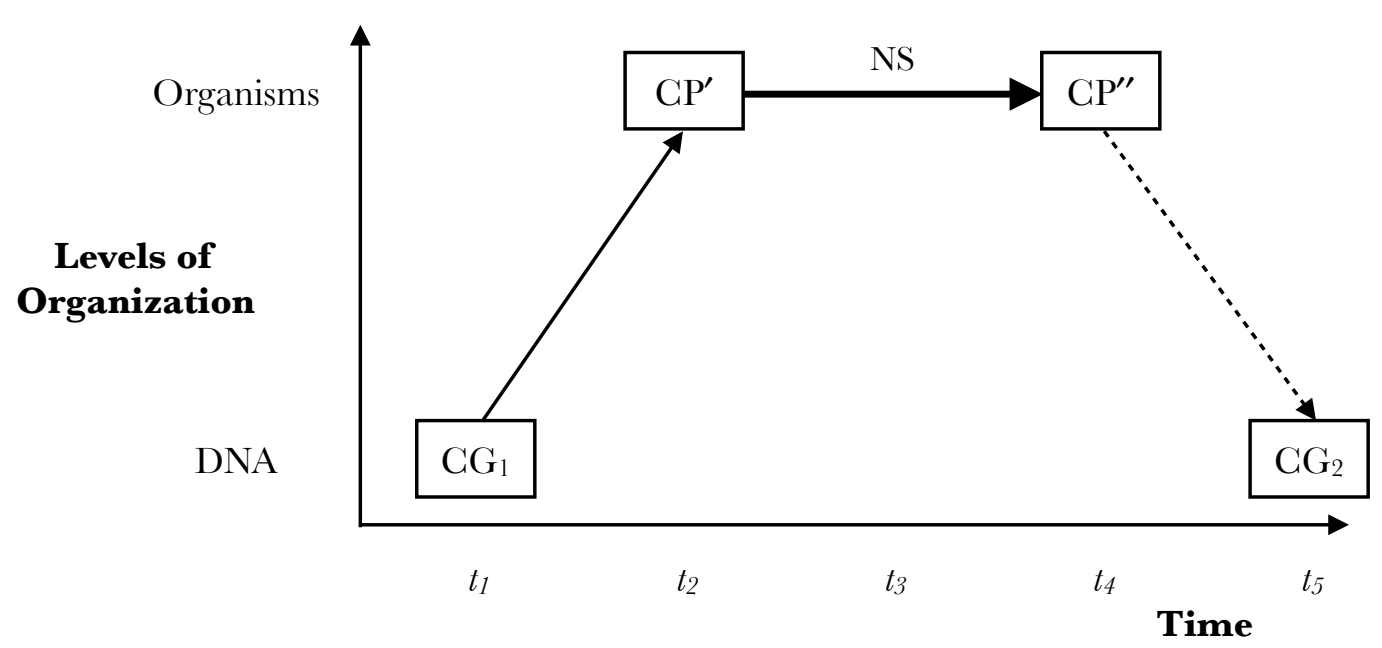

Figure 1 - A particular genetic configuration $\left(\mathbf{C G}_{1}\right)$ in a population (plus epigenetic laws) constructs a particular kind of phenotypes $\left(\mathrm{CP}^{\prime}\right)$ (represented by the solid arrow in an upward direction). Some of these phenotypes, once developed, will be selected by natural selection (NS) according to their fitness (black wide arrow). The selected phenotypes $\left(\mathrm{CP}^{\prime \prime}\right)$ will be the ones that leave offspring and pass their specific genes (through genetic heredity laws) to the next generation (represented by the dashed arrow in a downward direction), producing the new particular genotypic configuration $\left(\mathbf{G G}_{2}\right)$. This new configuration, in turn, will construct the new phenotypes. ${ }^{11}$ 


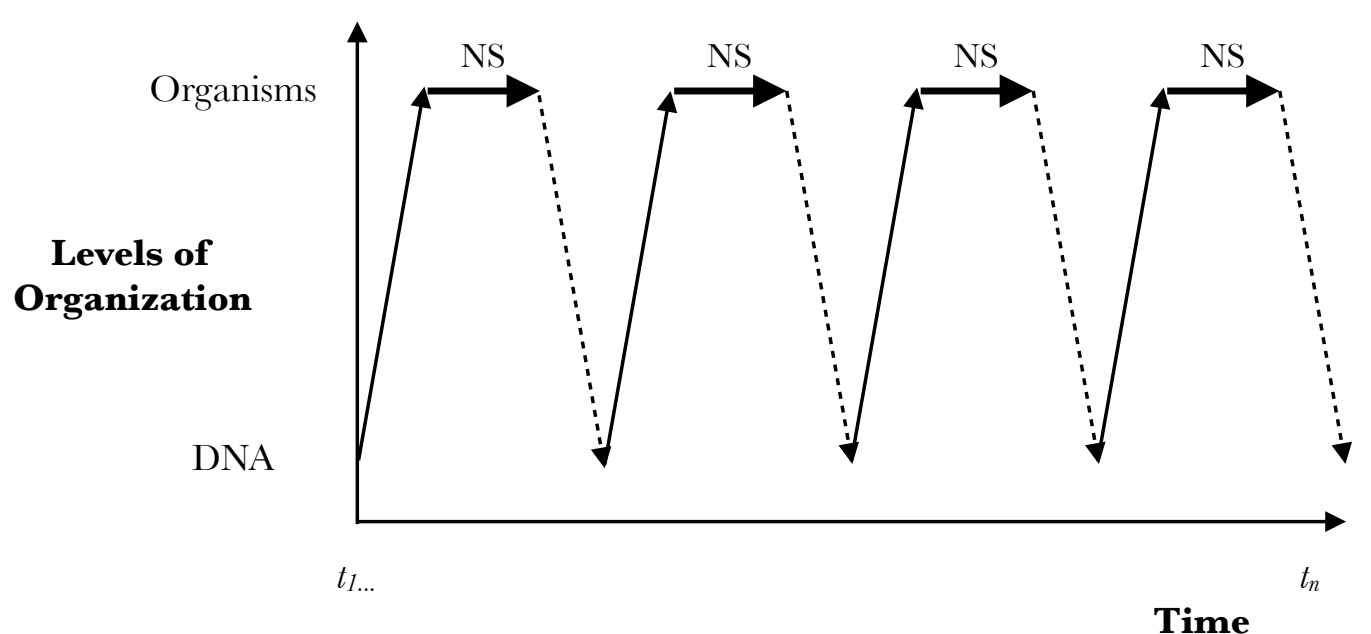

Figure 2 - Iteration of the process depicted in Figure 1. This illustrates a sequence of several generations of organisms causally linked through reproduction. Solid arrows represent the construction, depending on the genotypic configuration at a time $t_{x}$, of particular phenotypes, in a typical process of upward causation. Any single phenotype produced will, at the organismal level, interact with other phenotypes and the environment (feeding, mating, etc.), being an object of natural selection according to fitness (black horizontal arrows). Selection chooses which phenotypes will pass their genes to the next generation(s). Dashed arrows represent this downward influence of selection, at time $t_{y}$, on the arrangement of the DNA that will construct the new phenotypes.

can, in this way, affect the kinds and distribution of DNA templates leading to the generation of complex morphology. ${ }^{12}$

\section{Conclusion}

We have argued that the process of natural selection occurs in two steps: the blind (though neither isotropic nor equiprobable) generation of variation (through mutation or recombination, for example), and the subsequent (non-random) selection of this variation through differential replication. In this conception, both negative and positive views of natural selection are combined. But it is also possible to state that events of (negative) selection, if taken cumulatively, are a positive evolutionary factor - selection influences the generation of variation as well as the sorting of which variants increase in frequency. By choosing which phenotypes reproduce, natural selection affects the genetic configuration of the next generation(s). By influencing genetic configurations, selection positively affects the sources of variation. Recalling Neander's argument, if we consider only isolated and unrelated sequences of random-variation/selection events then we do not explain the particular configuration of gene sequences that leads to complex adaptations. On the other hand, if we focus on the relationships of numerous gene sequences interconnected and causally channeled by the downward influence of selection that reproduction allows, we explain how, in this strict sense, natural selection affects the generation of form and the evolutionary direction it takes. Downward causation successfully explains the type of multilevel causation operating in the natural selection process, while capturing the cumulative and creative action of the latter. For this reason it is useful to incorporate this type of causation in any definition of natural selection that seeks to explain the creative power it possesses. This reformulation of the concept of natural selection is based on the inclusion of the concept of downward causation. We believe our proposal addresses not only Darwin's intuition that natural selection was a creative force, but simultaneously strengthens and advances the work of post-Darwinian authors who have addressed the same issue. By highlighting the mutual co-determination between levels of organization in the process of natural selection we have recovered and articulated a multilevel perspective that is absent from previous discussions. Ayala's, Neander's, and Dobzhansky's analyses are focused on the genetic level, which, in our 
opinion, is just a (correct) part of the story. Needless to say, our work reflects a pluralistic spirit regarding causation. As Hitchcock $(2003,1)$ has recently said, "The goal of a philosophical account of causation should not be to capture the causal relation, but rather to capture the many ways in which the events of the world can be bound together."

Finally, we do not intend to argue that natural selection is the only factor that determines the direction taken by organic forms in their evolution. There are other equally important factors, such as developmental constraints or self-organization. These and other evolutionary factors, in conjunction with natural selection, come together as a plurality of positive causes that give direction to the evolution of form. It is the task of the newly created discipline of Evo-Devo to discriminate, in each particular morphological case, whether a trait obeys one factor more than another. The generation of organismal form is a complex process and that is why we think multilevel approaches that take into account diverse levels of organization, multiple directions of causation, and different time scales offer better prospects for understanding it.

\section{Literature cited}

Amundson, R. 2005. The Changing Role of the Embryo in Evolutionary Thought: The Roots of EvoDevo. New York: Cambridge University Press.

Andersen, P.B., Emmeche, C., Finnemann, N.O. and R.V. Christiansen. (eds) 2000. Downward Causation. Minds, Bodies, Matter. Aarhus: Aarhus University Press.

Ariew, A. 2003. Natural selection doesn't work that way: Jerry Fodor vs. Evolutionary Psychology on gradualism and saltationism. Mind \& Language 18: 478-483.

Ayala, F. 1970. Teleological explanations in evolutionary biology. Philosophy of Science 37: 1-15.

Ayala, F. 1993. Darwin's revolution. In: Creative Evolution?! Ed. by J. Campbell and J. Schopf. Boston: Jones and Bartlett.

Ayala, F. 1999 Adaptation and novelty: teleological explanations in evolutionary biology. History and Philosophy of the Life Sciences 21: 3-33.

Bedau, M. 2008. Downward causation and autonomy in weak emergence. In: Emergence: Contemporary Readings in Philosophy and Science. Ed. by M. Bedau and P. Humphreys. Cambridge, MA: MIT Press.

Bickhard, M. and D.T. Campbell. 2003. Variations in variation and selection: the ubiquity of the variationand-selective retention ratchet in emergent organizational complexity. Foundations of Science 8: 215-282.

Campbell, D.T. 1974. Downward causation in hierarchically organized biological systems. In: Studies in the Philosophy of Biology: Reduction and Related Problems. Ed. by F. Ayala and T. Dobzhansky. Londres: MacMillan.

Darwin, C. 1859. The Origin of Species. London: John Murray.

Dawkins, R. 1986. The Blind Watchmaker. London: Longmans.

Dowe, P. and P. Noordhof. 2004. Introduction. In: Cause and Chance. Causation in an Indeterministic World. Ed. by P. Dowe and P. Noordhof. New York: Routledge.

Dobzhansky, T. 1974. Chance and creativity in evolution. In: Studies in the Philosophy of Biology: Reduction and Related Problems. Ed. by F. Ayala and T. Dobzhansky. Londres: MacMillan.

El-Hani, C. and C. Emmeche. 2000. On some theoretical grounds for an organism-centered biology: property emergence, supervenience, and downward causation. Theory in Bioscience 119: 234-275.

Endler, J. 1986. Natural Selection in the Wild. Princeton: Princeton University Press.

Forber, P. 2005. On the explanatory roles of natural selection. Biology \& Philosophy 20: 329-342.

Gilbert, S., Opitz, J.M. and R.A. Raff. 1996. Resynthesizing evolutionary and developmental biology. Developmental Biology 173: 357-372.

Godfrey-Smith, P. 2009. Darwinian Populations and Natural Selection. New York: Oxford University Press.

Gould, S.J. 1999. Darwin and Paley meet the invisible hand. Natural History 99: 8-16.

Gould, S.J. 2002. The Structure of Evolutionary Theory. Cambridge, MA: The Belknap Press of Harvard University Press. 
Hamburger, V. 1980. Embryology and the Modern Synthesis in evolutionary theory. In: The Evolutionary Synthesis: Perspectives on the Unification of Biology. Ed. by E. Mayr and W. Provine. Cambridge, MA: Harvard University Press.

Hitchcock, C. 2003. Of Humean bondage. British Journal for the Philosophy of Science 54: 1-25.

Hodge, M.J.S. 1987. Natural selection as a causal, empirical, and probabilistic theory. In: The Probabilistic Revolution. Ed. by L. Kruger, G. Gigerenzer and M.S. Morgan. Cambridge, MA: MIT Press.

Jacob, F. 1977. Evolution and tinkering. Science 196: 1161-1166.

Juarrero. A. 1999. Dynamics in Action: Intentional Behavior as a Complex System. Cambridge, MA: MIT Press.

Kim, J. 1992. Downward causation in emergentism and nonreductive physicalism. In: Emergence or Reduction? Prospects for Nonreductive Physicalism. Ed. by A. Beckermann, H. Flohr, and J. Kim. Berlin: de Gruyter.

Kirschner, M. and J. Gerhart. 2005. The Plausibility of Life: Resolving Darwin's Dilemma. New Haven and London: Yale University Press.

Lewontin, R.C. The Genetic Basis of Evolutionary Change. New York: Columbia University Press.

Matthen, M. 1999. Evolution, Wisconsin style: selection and explanation of individual traits. British Journal for the Philosophy of Science 50: 143-150.

Matthen, M. 2003 Is sex really necessary? And other questions for Lewens. British Journal for the Philosophy of Science 54: 297-308.

Mayr, E. 1961. Cause and effect in biology. Science 134: 1501-1506.

Mayr, E. 1994. Response to John Beatty. Biology \& Philosophy 9: 357-358.

Mayr, E. 2002. What Evolution Is. New York: Basic Books

McLaughlin, P. [manuscript in preparation]. The Arrival of the Fittest.

Mitchell, S. 2009. Unsimple Truths: Science, Complexity, and Policy. Chicago: University of Chicago Press.

Müller, G. and S. Newman. 2003. Origination of organismal form: the forgotten cause in evolutionary biology. In: Origination of Organismal Form: Beyond the Gene in Developmental and Evolutionary Biology. Ed. by G.B. Müller and S. Newman. Cambridge, MA: MIT Press.

Nanay, B. 2005. Can cumulative selection explain adaptation? Philosophy of Science 72: 1099-1104.

Neander, K. 1988. What does natural selection explain? Philosophy of Science 55: 422-426.

Neander, K. 1995a. Pruning the tree of life. British Journal for the Philosophy of Science 46: 59-80.

Neander, K. 1995b. Explaining complex adaptations: a reply to Sober's reply to Neander. British Journal for the Philosophy of Science 46: 583-87.

Noble, D. 2006. The Music of Life: Biology Beyond Genes. New York: Oxford University Press.

Ohno, S. 1970. Evolution by Gene Duplication. Berlin: Springer Verlag.

Okasha. S. 2006. Evolution and the Levels of Selection. New York: Oxford University Press.

Ospovat, D. 1981. The Development of Darwin's Theory. New York: Cambridge University Press.

Paley, W. 1836 [1802]. Natural Theology. Londres: Charles Knight.

Plutynski, A. 2008. Explaining how and explaining why: developmental and evolutionary explanations of dominance. Biology \& Philosophy 23: 363-381.

Popper, K. 1978. Natural selection and the emergence of mind. Dialectica 32: 339-335.

Pust, J. 2004. Natural selection and the traits of individual organisms. Biology \& Philosophy 19: 765-79.

Ruse, M. 1979. The Darwinian Revolution. Chicago: University of Chicago Press.

Saunders, P.T. 1989. The evolution of form and pattern. Leonardo 22: 33-38.

Shapiro, L. and E. Sober. 2008. Epiphenomenalism: The Do's and the Don'ts. In: Studies in Causality: Historical and Contemporary. Ed. by G. Wolters and P. Machamer. Pittsburgh: University of Pittsburgh Press.

Sober, E. 1993 [1984]. The Nature of Selection. Chicago: University of Chicago Press.

Sober, E. 1995. Natural selection and distributive explanation: a reply to Neander. British Journal for the Philosophy of Science 46: 384-397.

Sober, E. 1993. Philosophy of Biology. Boulder, CO: Westview Press.

Soto, A.M. and C. Sonnenschein. 2005. Emergentism as a default: cancer as a problem of tissue organization. Journal of Bioscience 30: 103-118. 
Sperry, R.W. 1986. Macro- versus micro-determinism. Philosophy of Science 53: 265-270.

Suppes, P. 1970. Probabilistic Theory of Causality. Amsterdam: North-Holland Publishing Company.

Vrba, E. and N. Eldredge. 1984. Individuals, hierarchies and processes: towards a more complete evolutionary theory. Paleobiology 10:146-171.

Walsh, D. 1998. The scope of selection: Sober and Neander on what natural selection explains. Australasian Journal of Philosophy 76: 250-264.

West-Eberhard, M.J. 2003. Developmental Plasticity and Evolution. New York: Oxford University Press.

\section{Notes}

1. Neander (1995a) dubbed the two views on selection the 'negative view' and the 'positive view.' Pust (2004) divides the positions this way: Sober, Walsh, Lewens, and himself as supporters of the negative view, Neander and Matthen as defenders of the positive view. Nowadays, we can include the philosophers Forber, Nanay, GodfreySmith, and McLaughlin in the positive group. Matthen (2003) calls the negative view 'Anti-individualism' and the positive view 'Individualism.'

2. Two things are worth noting here. First, we agree with McLaughlin (manuscript in preparation) that all the philosophical treatments post-Sober that defend the negative view are mere refinements of Sober's original points, not substantial additions. Second, the issue of negative vs. positive views reaches beyond this philosophical debate because many biologists embrace the negative perspective without referring to the controversy (see below, Note 4 ). The controversy seems to invoke more than semantic issues as it deals with the nature of selection, its evolutionary role, and its epistemological status.

3. The criticisms of Sober could be justified partly by the fact that there is no clear treatment, at least in Sober's book (1993 [1984]) or in his reply to Neander (1995a), of the creative capacity of natural selection and its relationship to the distributive role he advocates. But it is also true that Sober mentions, in other places, that selection has a creative role in the evolution of complex traits (e.g., in his discussion of evolution vs. creationism; 1993, 36ff). However, it is not our aim to undertake an exegetical analysis of Sober's work on selection and evaluate whether its criticisms are justified. We simply aim to underscore the ideas that support the negative view and point out some of the consequences of taking these too literally, as many authors have done.

4. For example, Kirschner and Gerhart $(2005,13)$ argue that, "There are limits to what natural selection can do. We must remember that it acts merely as a filter, preserving some variants and rejecting others, natural selection does not create variation." West-Eberhard $(2003,197)$ agrees: "Research into selection and adaptation can tell us why a trait persisted and spread, but cannot tell where the trait came from." Saunders $(1989,33)$ in turn states that, "Selection can only retain, disseminate or remove variants that are already present in the population, it cannot, in itself, create new variants ... a theory so firmly focused on the destructive forces rather than the creative, cannot be but seriously incomplete." In the same vein, Gilbert et al. $(1996,368)$ affirm this perspective: "The role of natural selection in evolution, however, is seen to play less an important role. It is merely a filter for unsuccessful morphologies generated by development." Similarly, Endler $(1986,46)$ holds that, "Natural selection is not an explanation for adaptation, it only explains why and how, relatively, the best adaptations may increase in frequency ... Natural selection is the problem of the spread of new variants or new adaptations, not their origin." For a similar idea of negative natural selection, prior to Sober, see Ohno (1970).

5. The dictum that variation comes from mutation and natural selection distributes (chooses or reject) it in populations does not seem controversial. But some authors see this simple process as the base of an incomplete theory of evolution: if variations come from blind, isotropic, and random mutations, how can we explain shape and order in biological forms? Not by selection, because it just filters variations already present. They were, then, entirely shaped by other means (such as by developmental processes). And given that the Modern Synthesis left out development (Hamburger 1980), it is an incomplete theory of evolution (cf. Saunders 1989; Müller and Newman 2003).

6. The negative view is supported by compelling metaphors repeatedly associated with the process of selection (Neander 1995a; McLaughlin [manuscript in preparation]): selection acts as a 'filter' that eliminates less fit individuals (and their adaptations), or like a 'gardener's shears' that prune the tree of life. Both the variants to be filtered and the branches to be pruned exist prior to filtering or pruning, which means that natural selection (be it a filter or shears) plays no role in their origin. 
7. Ayala $(1970,1993)$ has illustrated the generation of novelty by natural selection with the following example, which focuses on the increase in the probability of successful gene combinations. Escherichia coli is a bacterium that needs the amino acid histidine to grow. If we put a few bacteria into a growth medium with 1 milliter of histidine, then these will multiply and, in a couple of hours, there will be two to three billion. In this bacterium, sporadic mutations conferring resistance to the antibiotic streptomycin occur on the order of one per one hundred million. If we add streptomycin to our bacterial culture, only twenty to thirty will survive, which, again, will multiply and within hours there will be twenty to thirty million, all of which will be resistant to this antibiotic. Likewise, sporadic mutations of $E$. coli that are able to grow without histidine occur on the order of four per one hundred million. If we transfer our new population of bacteria to a histidine-free medium, only a few hundred will survive. These in turn will multiply and in a few hours there will again be two to three billion, all resistant to streptomycin and also able to grow without histidine. Thus, while the probability of both mutations occurring spontaneously in the same bacterium is four in ten billion, natural selection has produced bacteria with these characteristics in just two steps. For Ayala, it is in this sense that selection can be called creative: natural selection gives rise to gene combinations that would otherwise be highly unlikely. "Natural selection has been compared to a sieve which retains the rarely arising useful and lets go the more frequently arising harmful mutations. Natural selection acts in that way, but it is much more than a purely negative process, for it is able to generate novelty by increasing the probability of otherwise extremely improbable genetic combinations. Natural selection is creative in a way" (Ayala 1970, 5). If we see the role of selection as merely distributive, then we will miss the creative role that is also present in this example.

8. As Godfrey-Smith (2009, 42), agreeing with the positive view of natural selection, has put it. Forber (2005) refines Neander's (1995a) argument in combination with a point made by Endler (1986). There are two important things worth noting about Forber's approach. First, he distinguishes between traits affected by a single factor and traits affected by multiple factors.

Consider two loci of a haploid organism, each with two competing alleles. Locus one has the alleles $A$ and $a$ while locus two has $B$ and $b$. Individuals with the $A B$ combination do much better than those with $A b, a B, a b$. If the $A B$ combination emerges, then it should quickly dominate the population. Now we ask how the $A B$ combination can evolve from a population made up of only $a b$ individuals. The role of selection becomes clear when we consider two contrasting cases, one with a selective gradient and one without. In the first case the combinations $A b$ and $a B$ do better than $a b$ but not $A B$; this represents the selective gradient. In the second, $A b$, $a B, a b$ all do equally well. The selective gradient in the first case makes it much more likely for the $A B$ combination to emerge (Forber 2005, 332).

According to Forber, we see the explanatory role of natural selection when a combination of factors affects a trait (in this case, the successful genetic combination). But he adds: "On Neander's view cumulative selection has a privileged explanatory status because previous trials affect the probability of later outcomes. But selection must do this in the right way by accumulating the multiple beneficial factors that affect a given trait" (2005, 334). We agree with Godfrey-Smith that Forber's approach is a refinement of Neander's argument, but we don't see anything substantially new - both Neander's example of the box with holes and numbered balls, and her reasoning about the necessity of the sequential order of mutations in the $G_{1}, G_{2}$, and $G_{3}$ case, implicitly refer to the combination of factors. The second thing we want to highlight in Forber's paper is his interesting reference to Endler (1986). Forber (2005, 341) uses Endler's argument about the capacity of selection for explaining the origins of traits. Neander's, Forber's, and Endler's arguments and reasoning are very similar in this respect. The curious thing is that Endler endorses Sober's negative view (see above, Note 4)!

9. Despite disagreements between philosophers on how probabilistic causation should be characterized, this approach is now (since Suppes 1970) common in philosophy and in science (Dowe and Noordhof 2004). We mention this because Ayala and Neander's (as well as Forber's) views of selection rest on probabilistic reasoning. They take selection as a factor that leads to the increase of the probability of the occurrence of particular events (i.e., it raises the probability of the appearance of fitter genetic combinations). For a precursor in the discussion of natural selection and probabilistic causation, see Hodge (1987). For an argument based on probability and counterfactuals that defends the positive view of selection, see Nanay (2005).

10. A very similar point was made by Jacob, three years later, in his classic "Evolution and Tinkering": "But natural selection does not act merely as a sieve eliminating detrimental mutations and favoring reproductions of beneficial ones, as is often suggested. In the long run, it integrates mutations, and orders them into adaptively coherent patterns adjusted over millions of years, and over millions of generations as a response to environmental challenges. It is natural selection that gives direction to changes, orients chance, and slowly, progressively produces more complex structures, new organs, and new species. Novelties come from previously unseen association of old material. To create is to recombine" (Jacob 1977, 1163). 
11. We are grateful to one of the referees for suggesting how to improve Figures 1 and 2 by reference to a graph from Lewontin $(1974,14)$. The suggestion was very useful.

12. It is worth mentioning that we do not use the common distinction between proximate and ultimate causes intentionally. Mayr (1961) introduced the dichotomy to distinguish evolutionary biology from molecular approaches, after which it was mainly used to refer to developmental processes as proximate and irrelevant to evolutionary explanations (Amundson 2005, 212). In his reply to Beatty, Mayr claims that there are two different kinds of causal processes in evolution and development - ultimate and proximate, respectively: to associate them is a hopeless task because they are unrelated (Mayr 1994, 357). But, as Amundson has pointed out, the proximateultimate distinction deals with kinds of explanations rather than with causation itself. He also argues that the dichotomy could be construed as a matter of degree: ontogeny can be "conceived as an intermediary stage between ancestral selection and an adult trait ... [as an] in-between point in the ultimate-proximate scale" (Amundson 2005, 204). We agree. If evolution and development are causally co-determined, a contrastive model is not useful to refer to the relationship between them. Biological causation, as is involved in the generation and shaping of organismal form, seems to be a complex matter that cannot be captured by Mayr's dichotomy. It should be noted that some defenders of the negative view of selection could be adopting the dichotomy as an ontological commitment; they see ultimate selective causes as unrelated to proximate developmental ones. See Plutynski (2008) for arguments to avoid the dichotomy and use wider causal frameworks in biology. For an analysis of the asymmetrical relationships between macro- and micro-causes, see Shapiro and Sober (2007).

\section{AGKNOWLEDGMENTS}

We are grateful to Maria Kronfeldner, Ulrich Krohs, two anonymous referees, and our editor for their valuable comments on the manuscript. Thanks also to the audiences at the Konrad Lorenz Institute (Altenberg, February 2009), the HPS seminar at Florida State University (Tallahassee, May 2009), and the Biolosophy meeting at the University of Bielefeld (December 2009) for useful discussions. MM wishes to thank the IIFs and the Coordinación de Humanidades at UNAM for financial support (2010). AM thanks the Proyecto SAF2009-13032-C02-01-Ministerio de Ciencia e Innovación (MICINN, Spain) and Proyecto Prometeo/2009/092-Generalitat Valenciana (Spain).

Copyright (C) 2011 Author(s).

This is an open-access article distributed under the terms of the Creative Commons Attribution-NonCommercial-NoDerivs license, which permits anyone to download, copy, distribute, or display the full text without asking for permission, provided that the creator(s) are given full credit, no derivative works are created, and the work is not used for commercial purposes.

ISSN 1949-0739 